\title{
Descriptive Review and Analysis of Immunoglobulin Utilization Management from 2,548 Prior Authorization Requests
}

\author{
Brian Tonkovic, PharmD, and Linda K. Rutishauser, RN
}

\begin{abstract}
BACKGROUND: Immunoglobulin (Ig) is a costly blood product prescribed as immune replacement or modulation therapy to treat a wide spectrum of medical conditions. While the FDA has approved Ig for a limited number of indications, there are multiple off-label uses that have demonstrated proven clinical benefit or are currently in various phases of clinical study. There are also diagnoses for which Ig is prescribed, but for which no evidence-based efficacy data are available. Many conditions for which Ig has been prescribed are extremely rare, and a controlled clinical trial is not logistically possible. These conditions may be denied insurance authorization because of limited medical evidence that supports the use of $\mathrm{Ig}$ in their treatment. This limited evidence consists of uncontrolled studies, case series and reports, and expert opinion. Limiting the use of Ig therapy to cases with the best supporting evidence may control health care expenditure, but it may also limit the potential body of knowledge from publications by physicians with experience in treating these conditions. Specialty pharmacy providers can collect longitudinal outcomes, and data from their publications could provide support for improved managed care criteria or areas of future medical research.
\end{abstract}

OBJECTIVES: To (a) assess levels of supporting evidence by diagnosis for a sample of Ig requests submitted through the managed care prior authorization (PA) process and (b) provide a descriptive review of such requests and resultant benefit coverage determinations by individual diagnosis, overarching diagnostic category, patient age group, and assessed levels of evidence.

METHODS: Through collaboration with 6 health plans, we obtained and analyzed 2,548 managed care PA requests for Ig therapy received between February 2008 and August 2012, as well as resulting benefit coverage determinations. A literature review of 7 established treatment guidelines and expert consensus statements was conducted and used to evaluate all indications for Ig treatment presented among obtained requests and to assign each request to 1 of 5 categorical levels of supporting evidence.

RESULTS: Of the 2,548 Ig requests reviewed, 1,467 (57.6\%) were found to be "supported" in the relevant literature; $830(32.6 \%)$ were designated "conditional"; 127 (5\%) were "lacking consensus"; 74 (2.9\%) were "currently not supported"; and $50(2 \%)$ were "undetermined." 0verall, 2,094 $(82.2 \%)$ of the requests were authorized by the health plans. Of the total requests, 1,633 $(64.1 \%)$ were for FDA-approved diagnostic indications, of which $1,393(85.3 \%)$ were authorized by the sampled health plans. The majority of requests for Ig were for treatment of neurological and immunological conditions. A total of 181 unique indications were identified, yet $833(32.7 \%)$ of the requests were for just 2 indications - common variable immunodeficiency and chronic inflammatory demyelinating polyneuropathy. Patients aged 45 to 64 years represented 1,318 (51.7\%) of all requests.

CONCLUSIONS: This analysis provides insight into the prescribed medical usage of Ig and the conditions for which it may be authorized through the managed care PA process. We identified 181 unique diagnostic indications for Ig, for which only 97 had established expert consensus. Although many diagnostic indications were identified, most requests were for indications with strong supporting evidence, and most of these were authorized by the health plans. The number of indications identified highlights the ongoing need for publications from physicians and specialty pharmacies with experience in treating these conditions in order to increase the body of knowledge surrounding use of this therapy.

J Manag Care Pharm. 2014;20(4):357-67

Copyright $\odot 2014$, Academy of Managed Care Pharmacy. All rights reserved.

\section{What is already known about this subject}

- Many conditions are reported in the literature for which immunoglobulin (Ig) has been evaluated as a therapeutic intervention.

- Experts have reviewed much of this literature and have prepared consensus statements on the uses of Ig that can serve as a clinical guide for this therapy.

- Many conditions for which Ig has been used are rare, and Ig usage to treat these conditions has been sparsely or only recently reported.

- There is a lack of published literature describing the current patterns of use for Ig in the United States.

What this study adds

- This study provides information on the range of conditions a U.S managed care organization may encounter for Ig therapy coverage verification

- This study highlights the varying degrees of supporting evidence for Ig therapy across a large number of diagnoses and the need for further research on Ig efficacy, especially in long-term care situations.

I mmunoglobulin (Ig) is an expensive blood product prepared from pooled donor plasma. The average annual cost of therapy has been estimated to range from $\$ 30,000$ to $\$ 90,000$ per patient, depending on dose, infusion time, length of treatment, and site of care. ${ }^{1}$ Ig is prescribed as replacement therapy in primary and secondary immune deficiencies and as immune modulation in autoimmune disorders. There are 6 indications with current U.S. Food and Drug Administration (FDA) approval for specific Ig products: primary immune deficiency, idiopathic thrombocytopenic purpura, chronic inflammatory demyelinating polyneuropathy (CIDP), multifocal motor neuropathy, chronic lymphocytic leukemia, and Kawasaki disease. ${ }^{2}$ Ig is also used to treat numerous off-label conditions that have been reported to represent $50 \%-80 \%$ of total $\mathrm{Ig}$ use. ${ }^{3}$ More than 160 of these other indications have been reported. ${ }^{4}$ This growing list of new potential indications 
for Ig may present a challenge to managed care organizations (MCOs), since a significant portion of requests may reflect conditions for which Ig, while clinically beneficial, is not currently FDA-approved, or for which insufficient research has been conducted to support or refute efficacy.

Prior authorization (PA) is a tool used by $82 \%$ of MCOs with the goal of managing Ig use by considering each case within the context of evidence-based treatment efficacy and positive outcomes. ${ }^{5}$ In a separate study, Leong et al. (2008) determined that interpretation of the medical evidence for Ig therapy among several types of organizations is inconsistent. ${ }^{4}$ The goal of our analysis was to characterize the indications for which Ig is currently being requested and to look at other factors, including patient demographics, supporting evidence, and benefit coverage outcomes, to enhance the body of knowledge surrounding this therapy. The PA process used by health plans provides a dataset uniquely suited to capturing this information, whereas a retrospective claims analysis would not have captured requests denied by the health plans.

\section{Methods}

\section{Description of Data}

An assessment of supporting evidence for Ig usage was conducted on requests submitted through the PA processes of 6 health plans, covering a combined total of approximately 15 million lives within the Central and Northeast regions of the United States. PA requests for Ig in all sites of care (home, physician office, and outpatient center) were collected, and identifying patient or requesting service provider information was omitted. The analysis included PA requests received by each MCO between February 2008 and August 2012, although data were not collected continuously from all collaborating plans throughout this time period. The files were collected electronically, and observations from collaborating health plans were combined into a single database. Data points collected included patient age, gender, treatment diagnosis, and initial benefit outcome (authorization or denial).

\section{Review of Published Evidence}

To assess the levels of evidence for Ig requests, we used 7 comprehensive guidelines with expert consensus recommendations specific to Ig uses. ${ }^{6-12}$ These sources are useful in evaluating Ig because the expert opinion and consensus provide balanced evidence assessments. Referencing these publications, we defined the following levels of evidence: "supported," "conditional," "lacking consensus," "currently not supported," and "undetermined." "Supported" conditions were defined as those diagnoses for which Ig is approved by the FDA and for which such use is similarly supported in consensus papers as a first-line treatment. When evaluating FDA approval status, we considered all ICD-9-CM (International Classification of Diseases, Ninth Revision, Clinical Modification) codes in the range of 279.0-279.13 as a primary immunodeficiency (see
Appendix, available in online article). Medical conditions for which Ig is considered an alternative or second-line treatment option, or not described uniformly among the cited guidelines and consensus papers, were designated "conditional," as were diagnoses for which there was insufficient evidence to support Ig over other interventions. For example, conditions in the study by Orange et al. (2006), which used descriptive evidence categories, including "probably beneficial" and "might provide benefit," were categorized as "conditional" in this analysis. ${ }^{11}$ Other sources used, such as the guidelines by Feasby et al. $(2007)^{6}$ and Anderson et al. (2007), ${ }^{9}$ provided more specific recommendations, suggesting that Ig should be considered only after other first-line agents have been tried and failed or are otherwise contraindicated. This scenario was also categorized as "conditional." Similarly, the guidelines referenced from the European Federation for Neurological Societies provided recommendations and practice points suggesting the role of Ig in therapy.

Rare diagnoses for which the use of Ig has been sparsely or only recently reported in the literature and for which guidelines have yet to be developed were designated "lacking consensus." Diagnoses for which Ig was considered to be ineffective or for which there existed insufficient evidence to support a beneficial effect in the cited guidelines were categorized as "currently not supported." In Orange et al. (2006), ${ }^{11}$ these conditions were categorized as "unlikely to be beneficial," and in other sources, recommendations were more specific, suggesting that the use of Ig was not recommended. Finally, some diagnosis data were not presented uniformly and could not be referenced against the consensus papers. This small fraction of requests was designated "undetermined." An important consideration is that recommendations from the sources cited in this study were based on the best available evidence at the time of publication. Although many of the conditions described in this analysis are rare, new evidence has possibly become available since these expert reviews were published, and that evidence may further support or refute the use of Ig for treatment of these conditions.

We reviewed each diagnostic indication in the data provided by health plans, aggregated the recommendations from the guidelines cited, and assigned each PA request an evidence grade from among the list of defined classifications. The purpose of assigning an evidence grade was to determine the overall levels of supporting evidence for the extensive number of conditions observed in this analysis. Those who prescribe Ig should review treatment guidelines for specific recommendations.

\section{Design of Review}

Our report is intended to be primarily descriptive, detailing Ig prescription and benefit review decisions based on diagnostic categories and on levels of evidence for Ig usage as per current guidelines. The intention is to expand current knowledge on the use of this therapy. 


\begin{tabular}{l|c|c|c}
\hline TABLE 1) & \multicolumn{3}{|c}{$\begin{array}{l}\text { Evidence Levels by Benefit } \\
\text { Coverage Outcome }\end{array}$} \\
\hline & $\begin{array}{c}\text { Health Plan } \\
\text { Benefits } \\
\text { Authorized } \\
\text { (n) }\end{array}$ & $\begin{array}{c}\text { Health Plan } \\
\text { Benefits } \\
\text { Denied } \\
\text { (n) }\end{array}$ & Total \\
\hline Suidence Level & 1,272 & 195 & 1,467 \\
\hline Conditional & 696 & 134 & 830 \\
\hline Lacking consensus & 62 & 65 & 127 \\
\hline Currently not supported & 30 & 44 & 74 \\
\hline Undetermined & 34 & 16 & 50 \\
\hline Total & 2,094 & 454 & 2,548 \\
\hline
\end{tabular}

Results

\section{FDA Status}

A total of 2,548 requests were reviewed, covering 181 unique diagnostic indications. Of these requests, 1,633 (64.1\%) were for current FDA-labeled indications, the majority of which1,393 (85.3\%) —were authorized by sampled health plans. Of the 915 (35.9\%) PA requests for non-FDA-approved indications, benefit coverage was authorized for $76.6 \%$.

\section{Evidence Levels}

Among the 2,548 requests evaluated, the following levels of supporting evidence were established: 1,467 (57.6\%) "supported," 830 (32.6\%) "conditional," 127 (5\%) "lacking consensus," 74 (2.9\%) "currently not supported," and 50 (2\%) "undetermined." Overall, 2,094 requests (82.2\%) were authorized by the health plans, and 454 (17.8\%) were denied. When the benefit authorization status was reviewed by levels of evidence, the following rates of approval were identified (Table 1): "supported" 1,272 (86.7\%), "conditional" 696 (83.9\%), "lacking consensus" 62 (83.8\%), "currently not supported" 30 $(40.5 \%)$, and "undetermined" 34 (68\%). For all evidence levels, the number of authorizations exceeded denials, except for those considered "currently not supported." Interestingly, only a few diagnostic indications were more frequently denied than authorized. Notably, within the "conditional" category, pediatric autoimmune neuropsychiatric disorder - a disorder lacking a unique ICD-9-CM code-had more denials than authorizations. Of the conditions "currently not supported," all requests for recurrent, spontaneous fetal loss, diabetic neuropathy, inclusion body myositis, and autism were denied.

\section{Diagnostic Category}

As expected, the majority of conditions for which Ig treatment was requested were immunologic (41.9\%) and neurologic (32.8\%). Hematologic indications, for which Ig is most often used to treat immune deficiency secondary to malignancy, were also quite common (14.8\%).

Of the 837 neurology requests, 660 (78.9\%) were authorized by the health plans, and 177 (21.1\%) were denied. Of the

\section{TABLE 2 Diagnostic Category by} Benefit Coverage Outcome

\begin{tabular}{l|c|c|c}
\hline Disease Category & $\begin{array}{c}\text { Health Plan } \\
\text { Benefits } \\
\text { Authorized } \\
\text { (n) }\end{array}$ & $\begin{array}{c}\text { Health Plan } \\
\text { Benefits } \\
\text { Denied } \\
\text { (n) }\end{array}$ & Total \\
\hline Immunology & 895 & 172 & 1,067 \\
\hline Neurology & 660 & 177 & 837 \\
\hline Hematology & 325 & 53 & 378 \\
\hline Rheumatology & 127 & 14 & 141 \\
\hline Solid organ transplantation & 54 & 3 & 57 \\
\hline Other & 20 & 22 & 42 \\
\hline Dermatology & 11 & - & 11 \\
\hline Infectious diseases & 1 & 2 & 3 \\
\hline Oncology & 1 & - & 1 \\
\hline Obstetrics/gynecology & - & 11 & 11 \\
\hline Total & 2,094 & 454 & 2,548 \\
\hline
\end{tabular}

neurology cases that were denied, the following levels of evidence were found: 55 (31.1\%) were for conditions "currently not supported" or "lacking consensus"; 52 (29.4\%) were "supported"; 55 (31.1\%) were "conditional"; and 15 (8.5\%) were "undetermined." Of the 1,067 immunology requests, 895 (83.9\%) were authorized, and 172 (16.1\%) were denied. Of the authorized immunology cases, the following levels of evidence were found: 737 (82.3\%) "supported," 149 (16.6\%) "conditional," 2 (0.22\%) "lacking consensus," and 7 (0.78\%) "currently not supported." However, 166 (96.5\%) of the immunology denials were for "supported" or "conditional" uses, suggesting that many requests did not fulfill coverage criteria established by the health plans. Other diagnostic categories represented $25.3 \%$ of total cases, of which $85 \%$ were authorized (Table 2).

\section{Diagnostic Indication}

Our data reflect a wide range of conditions for which Ig was requested, a total of 181 unique indications (Table 3). The most frequent diagnosis was common variable immunodeficiency (19.7\%). Other immune deficiency conditions included hypogammaglobulinemia (11\%) and selective immunoglobulin deficiencies (4.2\%). CIDP, a neurological condition that recently received FDA approval for treatment with Ig, represented $13 \%$ of all requests and received the second-highest number of treatment requests. Other neurological conditions included multiple sclerosis, myasthenia gravis, and multifocal motor neuropathy, with a combined representation of $11.7 \%$. Chronic lymphocytic leukemia, dermatomyositis, and immune thrombocytopenia purpura were among the other indications for which treatment requests were submitted.

\section{Age Group}

Of the top 3 diagnostic categories for all requests, $51.7 \%$ of all PA requests were for the group aged 45-64 years: 477 (57\%) 


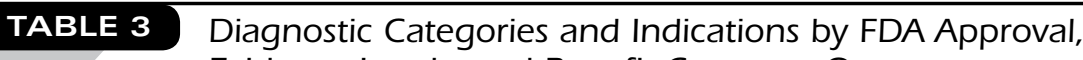
Evidence Levels, and Benefit Coverage Outcome

\begin{tabular}{|c|c|c|c|c|c|}
\hline Evidence Level & $\begin{array}{c}\text { FDA } \\
\text { Approval }\end{array}$ & Diagnosis & $\begin{array}{c}\text { Health Plan Benefits } \\
\text { Authorized (n) }\end{array}$ & $\begin{array}{l}\text { Health Plan Benefits } \\
\text { Denied (n) }\end{array}$ & Total \\
\hline \multirow[t]{25}{*}{ Supported } & no & Bone marrow transplant & 25 & 3 & 28 \\
\hline & no & Neonatal alloimmune thrombocytopenia & 4 & 1 & 5 \\
\hline & no & Lewis-Sumner syndrome & 4 & - & 4 \\
\hline & no & Myelodysplastic syndrome & 2 & - & 2 \\
\hline & no & Ataxia telangiectasia & 1 & - & 1 \\
\hline & no & Parvovirus-induced chronic anemia & 1 & - & 1 \\
\hline & no & Graft-versus-host disease & - & 1 & 1 \\
\hline & yes & Common variable immunodeficiency & 439 & 62 & 501 \\
\hline & yes & Chronic inflammatory demyelinating polyneuropathy & 297 & 35 & 332 \\
\hline & yes & Hypogammaglobulinemia & 230 & 50 & 280 \\
\hline & yes & Immune thrombocytopenic purpura & 101 & 4 & 105 \\
\hline & yes & Chronic lymphocytic leukemia & 53 & 12 & 65 \\
\hline & yes & Multifocal motor neuropathy & 44 & 17 & 61 \\
\hline & yes & X-linked agammaglobulinemia & 32 & 3 & 35 \\
\hline & yes & IgG subclass deficiency & 15 & 4 & 19 \\
\hline & yes & Severe combined immunodeficiency & 7 & - & 7 \\
\hline & yes & Combined immunity deficiency & 4 & 3 & 7 \\
\hline & yes & Digeorge's syndrome & 3 & - & 3 \\
\hline & yes & Hyper IgM syndrome & 3 & - & 3 \\
\hline & yes & Humoral immunity deficiency & 2 & - & 2 \\
\hline & yes & Wiskott-Aldrich syndrome & 1 & - & 1 \\
\hline & yes & Wegener's granulomatosis & 1 & - & 1 \\
\hline & yes & Chronic inflammatory sensory polyradiculoneuropathy & 1 & - & 1 \\
\hline & yes & Deficiency of humoral immunity, other & 1 & - & 1 \\
\hline & yes & Hyper IgE syndrome & 1 & - & 1 \\
\hline \multicolumn{3}{|l|}{ Supported total } & 1,272 & 195 & 1,467 \\
\hline \multirow[t]{27}{*}{ Conditional } & no & Acquired hypogammaglobulinemia & 115 & 23 & 138 \\
\hline & no & Multiple sclerosis & 87 & 13 & 100 \\
\hline & no & Myasthenia gravis with (acute) exacerbation & 83 & 7 & 90 \\
\hline & no & Dermatomyositis & 66 & 3 & 69 \\
\hline & no & Myasthenia gravis without (acute) exacerbation & 35 & 14 & 49 \\
\hline & no & Polymyositis & 40 & 1 & 41 \\
\hline & no & Guillain-Barré syndrome & 25 & 4 & 29 \\
\hline & no & Transplant/kidney: desensitization & 21 & - & 21 \\
\hline & no & Transplant rejection/kidney: AMR & 16 & - & 16 \\
\hline & no & Stiff-person syndrome & 10 & 4 & 14 \\
\hline & no & Secondary hypogammaglobulinemia & 5 & 5 & 10 \\
\hline & no & Pediatric autoimmune neuropsychiatric disorder & 4 & 6 & 10 \\
\hline & no & Systemic lupus erythematosus & 7 & 1 & 8 \\
\hline & no & Autoimmune hemolytic anemia & 6 & - & 6 \\
\hline & no & IgM monoclonal gammopathy & 2 & 2 & 4 \\
\hline & no & Pemphigus foliaceus & 4 & - & 4 \\
\hline & no & Brachial plexus lesions & 1 & 2 & 3 \\
\hline & no & Acute disseminated encephalomyelitis & 2 & 1 & 3 \\
\hline & no & Pemphigus vulgaris & 3 & - & 3 \\
\hline & no & Pure red cell aplasia & 1 & 1 & 2 \\
\hline & no & Bullous pemphigoid & 2 & - & 2 \\
\hline & no & Chronic urticaria & 1 & 1 & 2 \\
\hline & no & Severe myoclonic epilepsy in infancy & 1 & - & 1 \\
\hline & no & Bullous dermatosis & 1 & - & 1 \\
\hline & no & Fetal-neonatal alloimmune thrombocytopenia & 1 & - & 1 \\
\hline & no & Cerebellar ataxia & 1 & - & 1 \\
\hline & no & Cicatricial ocular pemphigoid & 1 & - & 1 \\
\hline
\end{tabular}




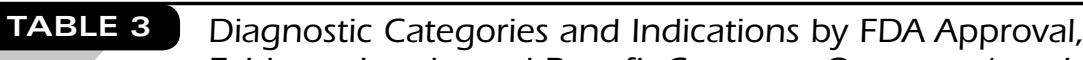
Evidence Levels, and Benefit Coverage Outcome (continued)

\begin{tabular}{|c|c|c|c|c|c|}
\hline Evidence Level & \begin{tabular}{|c|} 
FDA \\
Approval
\end{tabular} & Diagnosis & $\begin{array}{c}\text { Health Plan Benefits } \\
\text { Authorized (n) }\end{array}$ & $\begin{array}{l}\text { Health Plan Benefits } \\
\text { Denied (n) }\end{array}$ & Total \\
\hline & no & Polyneuritis & 1 & - & 1 \\
\hline & no & Intestinal infection C. difficile enteritis & - & 1 & 1 \\
\hline & no & Chronic parvoviral B19 infection & 1 & - & 1 \\
\hline & no & Thrombocytopenic purpura & 1 & - & 1 \\
\hline & no & Rasmussen encephalitis & 1 & - & 1 \\
\hline & no & Monoclonal gammopathy of unknown significance & 1 & - & 1 \\
\hline & no & Recurrent $C$. difficile colitis & - & 1 & 1 \\
\hline & no & Agnogenic myeloid metaplasia & 1 & - & 1 \\
\hline & yes & Selective Ig deficiency & 76 & 31 & 107 \\
\hline & no & Unspecified immune deficiency & 28 & 5 & 33 \\
\hline & yes & Specific antibody deficiency & 29 & 4 & 33 \\
\hline & yes & Selective antibody deficiency with normal Ig & 16 & 4 & 20 \\
\hline \multicolumn{3}{|l|}{ Conditional total } & 696 & 134 & 830 \\
\hline \multirow[t]{40}{*}{ Lacking consensus } & no & Sjogren's syndrome & 8 & 1 & 9 \\
\hline & no & Small fiber neuropathy & 4 & 3 & 7 \\
\hline & no & Mononeuritis multiplex & 3 & 4 & 7 \\
\hline & no & Scleroderma & 2 & 3 & 5 \\
\hline & no & Neuromyelitis optica & 4 & - & 4 \\
\hline & no & Encephalopathy & - & 4 & 4 \\
\hline & no & Autonomic neuropathy & 1 & 3 & 4 \\
\hline & no & Autoimmune retinopathy & 1 & 2 & 3 \\
\hline & no & Optic neuritis & 1 & 2 & 3 \\
\hline & no & Susac's syndrome & 3 & - & 3 \\
\hline & no & Neurosarcoidosis & 2 & 1 & 3 \\
\hline & no & Autonomic small fiber neuropathy & - & 3 & 3 \\
\hline & no & Protein-losing enteropathy & 1 & 1 & 2 \\
\hline & no & Limbic encephalitis & 1 & 1 & 2 \\
\hline & no & BK virus nephropathy & 2 & - & 2 \\
\hline & no & Netherton syndrome & 1 & 1 & 2 \\
\hline & no & Pancytopenia & 1 & 1 & 2 \\
\hline & no & Axonal polyneuropathy & 1 & 1 & 2 \\
\hline & no & Lyme disease & - & 2 & 2 \\
\hline & no & Antisulfatide antibody positive neuropathy & 1 & 1 & 2 \\
\hline & no & Demyelinating disease & - & 2 & 2 \\
\hline & no & Opsoclonus myoclonus & 2 & - & 2 \\
\hline & no & Necrotizing myopathy & 2 & - & 2 \\
\hline & no & Inflammatory neuropathy & 1 & 1 & 2 \\
\hline & no & Eosinophilic fasciitis & - & 1 & 1 \\
\hline & no & Pansclerotic morphea & 1 & - & 1 \\
\hline & no & Norovirus & 1 & - & 1 \\
\hline & no & Hereditary hemochromatosis & - & 1 & 1 \\
\hline & no & Autoimmune-mediated neuropathy & - & 1 & 1 \\
\hline & no & Hypocomplementemic urticarial vasculitis & - & 1 & 1 \\
\hline & no & Torsion dystonia & - & 1 & 1 \\
\hline & no & IgG monoclonal gammopathy & - & 1 & 1 \\
\hline & no & Churg-Strauss syndrome & 1 & - & 1 \\
\hline & no & Inflammatory demyelination & 1 & - & 1 \\
\hline & no & Parvovirus B polyarthropathy & - & 1 & 1 \\
\hline & no & Inflammatory myopathy & 1 & - & 1 \\
\hline & no & Relapsing transverse myelitis & - & 1 & 1 \\
\hline & no & Transverse myelitis & - & 1 & 1 \\
\hline & no & Hashimoto encephalopathy & - & 1 & 1 \\
\hline & no & Unspecified disease of spinal cord & - & 1 & 1 \\
\hline
\end{tabular}


TABLE 3 Diagnostic Categories and Indications by FDA Approval, Evidence Levels, and Benefit Coverage Outcome (continued)

\begin{tabular}{|c|c|c|c|c|c|}
\hline Evidence Level & $\begin{array}{c}\text { FDA } \\
\text { Approval }\end{array}$ & Diagnosis & $\begin{array}{c}\text { Health Plan Benefits } \\
\text { Authorized (n) }\end{array}$ & $\begin{array}{l}\text { Health Plan Benefits } \\
\text { Denied (n) }\end{array}$ & Total \\
\hline & no & Toxic neuropathy & - & 1 & 1 \\
\hline & no & Vasculitis & 1 & - & 1 \\
\hline & no & Chronic maxillary sinusitis & 1 & - & 1 \\
\hline & no & Autoimmune disorder & - & 1 & 1 \\
\hline & no & Autoimmune sensory polyradiculopathy & 1 & - & 1 \\
\hline & no & Autoimmune myelopathy & 1 & - & 1 \\
\hline & no & Parainfluenza virus & 1 & - & 1 \\
\hline & no & Autoimmune pancytopenia & 1 & - & 1 \\
\hline & no & Primary carnitine deficiency & - & 1 & 1 \\
\hline & no & Lymphadenopathy & - & 1 & 1 \\
\hline & no & Pulmonary hemosiderosis & 1 & - & 1 \\
\hline & no & BK virus & - & 1 & 1 \\
\hline & no & Sarcoidosis & - & 1 & 1 \\
\hline & no & Autoimmune autonomic ganglioneuropathy & - & 1 & 1 \\
\hline & no & Fibromyalgia & - & 1 & 1 \\
\hline & no & Blau syndrome & 1 & - & 1 \\
\hline & no & Hemochromatosis & 1 & - & 1 \\
\hline & no & Cervical radiculitis & - & 1 & 1 \\
\hline & no & Tourette's syndrome & - & 1 & 1 \\
\hline & no & Chronic lung disease & 1 & - & 1 \\
\hline & no & Hemophagocytic lymphohistiocytosis & 1 & - & 1 \\
\hline & no & Neutropenia & - & 1 & 1 \\
\hline & no & Vasculitic neuropathy & - & 1 & 1 \\
\hline & no & Inflammatory polyartropathy & - & 1 & 1 \\
\hline & no & X-linked inhibitor of apoptosis & - & 1 & 1 \\
\hline & no & Intestinal lymphangiectasia & 1 & - & 1 \\
\hline & no & Langerhans cell histiocytosis & 1 & - & 1 \\
\hline & yes & Transient hypogammaglobulinemia of infancy & 1 & 2 & 3 \\
\hline & yes & Selective IgM deficiency & 1 & 1 & 2 \\
\hline \multicolumn{3}{|l|}{ Lacking consensus total } & 62 & 65 & 127 \\
\hline \multirow[t]{23}{*}{ Currently not supported } & no & Recurrent, spontaneous fetal loss & - & 11 & 11 \\
\hline & no & Amyotrophic lateral sclerosis & 1 & 4 & 5 \\
\hline & no & Diabetic neuropathy & - & 5 & 5 \\
\hline & no & Inclusion body myositis & - & 4 & 4 \\
\hline & no & Progressive multiple sclerosis & 1 & 3 & 4 \\
\hline & no & Transplant rejection/kidney: cellular & 2 & 1 & 3 \\
\hline & no & Transplant rejection/heart: AMR & 3 & - & 3 \\
\hline & no & Autism & - & 3 & 3 \\
\hline & no & Secondary progressive multiple sclerosis & 2 & 1 & 3 \\
\hline & no & Transplant rejection/lung: AMR & 2 & - & 2 \\
\hline & no & Transplant rejection/heart: cellular & - & 2 & 2 \\
\hline & no & Transplant/heart: desensitization & 2 & - & 2 \\
\hline & no & Dravet syndrome & 1 & - & 1 \\
\hline & no & Transplant rejection/liver: unspecified & 1 & - & 1 \\
\hline & no & Antiphospolipid syndrome & - & 1 & 1 \\
\hline & no & Transplant/lung: desensitization & 1 & - & 1 \\
\hline & no & Transplant rejection/lung: unspecified & 1 & - & 1 \\
\hline & no & Clinically isolated syndrome & - & 1 & 1 \\
\hline & no & Epilepsy & - & 1 & 1 \\
\hline & no & Paraneoplastic neuropathy & - & 1 & 1 \\
\hline & no & Intractable seizure disorder & 1 & - & 1 \\
\hline & no & Cystic fibrosis & 1 & - & 1 \\
\hline & no & Leukodystrophy & - & 1 & 1 \\
\hline
\end{tabular}


TABLE 3 Diagnostic Categories and Indications by FDA Approval, Evidence Levels, and Benefit Coverage Outcome (continued)

\begin{tabular}{|c|c|c|c|c|c|}
\hline Evidence Level & $\begin{array}{c}\text { FDA } \\
\text { Approval }\end{array}$ & Diagnosis & $\begin{array}{c}\text { Health Plan Benefits } \\
\text { Authorized (n) }\end{array}$ & $\begin{array}{c}\text { Health Plan Benefits } \\
\text { Denied (n) }\end{array}$ & Total \\
\hline & no & Progressive muscle weakness & 1 & - & 1 \\
\hline & no & Aplastic anemia & 1 & - & 1 \\
\hline & no & Myoclonic astatic epilepsy & - & 1 & 1 \\
\hline & no & von Willebrand's disease & 1 & - & 1 \\
\hline & no & Paraneoplastic encephalitis & - & 1 & 1 \\
\hline & no & Paraneoplastic encephalopathy & 1 & - & 1 \\
\hline & yes & Selective IgA deficiency & 7 & 3 & 10 \\
\hline \multicolumn{3}{|c|}{ Currently not supported total } & 30 & 44 & 74 \\
\hline \multirow[t]{18}{*}{$\underline{\text { Undetermined }}$} & no & Peripheral neuropathy & 8 & 5 & 13 \\
\hline & no & Polyneuropathy & 6 & 1 & 7 \\
\hline & no & Myopathy, unspecified & 4 & 3 & 7 \\
\hline & no & Thrombocytopenia & 6 & 1 & 7 \\
\hline & no & Myositis, unspecified & 1 & 1 & 2 \\
\hline & no & Motor neuron disease & - & 2 & 2 \\
\hline & no & Neuropathy & 1 & - & 1 \\
\hline & no & Progressive demyelinating polyneuropathy & 1 & - & 1 \\
\hline & no & Polyradiculopathy & - & 1 & 1 \\
\hline & no & Paraplegia & 1 & - & 1 \\
\hline & no & Primary sensory radiculoneuropathy & 1 & - & 1 \\
\hline & no & Sensory neuropathy & - & 1 & 1 \\
\hline & no & Sensory ganglionopathy & 1 & - & 1 \\
\hline & no & Weakness and numbness & - & 1 & 1 \\
\hline & no & Myasthenic-like syndrome & 1 & - & 1 \\
\hline & no & Parotid adenocarcinoma & 1 & - & 1 \\
\hline & no & Anti-synthetase syndrome & 1 & - & 1 \\
\hline & no & Parvovirus B19 & 1 & - & 1 \\
\hline Undetermined total & & & 34 & 16 & 50 \\
\hline Overall total & & & 2,094 & 454 & 2,548 \\
\hline
\end{tabular}

neurology, 517 (48.5\%) immunology, and 211 (55.8\%) hematology (Table 4). In this cohort, 1,066 requests $(80.9 \%)$ were authorized, and 252 (19.1\%) were denied (Table 5). There were 308 requests for patients aged $\leq 18$ years, 270 (87.7\%) of which were authorized. The highest authorization rates were observed for the groups aged $\leq 2$ years and $13-18$ years, with $95.2 \%$ and $94.2 \%$ of requests authorized, respectively. The lowest rates of authorization were found in the group aged $\geq 80$ years, with $75 \%$ of requests authorized. Reviewing the other age groups in the top 3 diagnostic categories, the highest percentages of benefit denials were as follows: hematology 35 (16.6\%) for the group aged 45-64 years, neurology 2 (33.3\%) for the group aged 2-5 years, and immunology 104 (20.1\%) for the group aged $45-64$ years.

\section{Discussion}

Our data describe the patterns of Ig use identified through the PA process commonly used by MCOs. We identified a heterogeneous group of diagnostic indications reflecting the growing list of indications for which Ig is currently consid- ered. We observed that $35.9 \%$ of all requests were for FDA off-label indications. The FDA does not restrict the clinical use of off-label products in clinical practice. Importantly, an FDA off-label designation does not suggest that these indications are inappropriate, and clinicians may choose to prescribe Ig for indications for which a benefit is perceived. For many of these off-label conditions, Ig has become an effective standard of care supported by evidence-based medicine. In these cases, we noted a $23.4 \%$ rate of denial, suggesting that there may be a significant number of patients unable to start on therapy for conditions that respond to Ig. Many of the off-label conditions in our analysis were conditions for which the supporting evidence was limited to case studies or series and expert opinion. These conditions may be excluded from payer medical policies. For this reason, there may be many patients for whom therapy was considered by the physician but not requested, under the assumption that it would not be a covered benefit.

There is limited research regarding Ig insurance authorization for intravenous use (IVIg) in terms of supporting evidence 
Descriptive Review and Analysis of Immunoglobulin Utilization Management from 2,548 Prior Authorization Requests

\section{TABLE 4 Diagnostic Categories by Age Group in Years}

\begin{tabular}{|c|c|c|c|c|c|c|c|c|c|}
\hline Disease Category & $\leq 2(\mathrm{n})$ & $2-5(n)$ & $6-12(n)$ & $13-18(n)$ & $19-44(n)$ & 45-64 (n) & $65-79(n)$ & $\geq 80(\mathrm{n})$ & Unknown (n) \\
\hline Dermatology & - & - & - & - & 1 & 8 & 1 & - & 1 \\
\hline Hematology & 2 & 14 & 11 & 9 & 79 & 211 & 45 & 4 & 3 \\
\hline Immunology & 18 & 27 & 90 & 56 & 312 & 517 & 41 & 4 & 2 \\
\hline Infectious diseases & - & - & - & - & - & 3 & - & - & - \\
\hline Neurology & - & 6 & 23 & 10 & 218 & 477 & 90 & 8 & 5 \\
\hline Obstetrics/gynecology & - & - & - & - & 10 & - & - & - & 1 \\
\hline Oncology & - & 1 & - & - & - & - & - & - & - \\
\hline Other & - & 4 & 9 & 2 & 13 & 13 & 1 & - & - \\
\hline Rheumatology & - & 4 & 7 & 6 & 55 & 62 & 7 & - & - \\
\hline Solid organ transplantation & 1 & 2 & 3 & 3 & 17 & 27 & 4 & - & - \\
\hline Total & 21 & 58 & 143 & 86 & 705 & 1,318 & 189 & 16 & 12 \\
\hline
\end{tabular}

for efficacy of treatment. Several authors have described the conditions for which Ig is used in treatment. Hanna et al. (2003), via a retrospective analysis of IVIg use across a 10-hospital Canadian system, evaluated the indications for which IVIg was requested..$^{13}$ The authors referenced the 1997 and 1999 Canadian Consensus Guidelines for their evaluations. They identified 90 unique indications, 40 of which were determined to be "not supported" by current clinical evidence, and another 24 defined as requiring "future research." Although the "not supported" and "future research" categories represented 71\% of all indications for which Ig was requested, those categories applied to only $6 \%$ of the patients, suggesting that only a few patients received empiric therapy for those diagnoses. ${ }^{13}$ We note that additional reports and guidelines have been published since this study was conducted that may provide evidence to support some of the indications identified in the study. We observed a comparable trend in our data. Of the 181 diagnostic indications found in our 2,548 sampled requests, 117 indications (64.6\%) were categorized as "lacking consensus," "currently not supported," or "undetermined," but these appeared in only 251 (9.8\%) of the total requests. This small percentage suggests that although there are many diagnostic indications for Ig, most patients receive therapy for "supported" uses. Also as described in our own findings, Ig was used most prevalently in neurologic and hematologic diagnostic categories. In the study by Hanna et al., 4 indications-immune thrombocytopenia, CIDP, primary immunodeficiency, and hypogammaglobulinemia-comprised $72 \%$ of IVIg requests. ${ }^{13}$

Pendergrast et al. (2005) evaluated the use of IVIg in 429 Canadian hospitals during a period of IVIg shortages to assess changes in prescribing behaviors. ${ }^{14}$ Similar to the findings reported here and in the Hanna et al. study, the use of IVIg in hematology and neurology was the most common, representing $50 \%$ of the indications. The authors concluded that $75 \%$ of the patients treated had an indication approved by at least 1 expert body from 1995 to $2000 .{ }^{14}$ The study identified the 5 most common indications as immune thrombocytopenic purpura, hypogammaglobulinemia, Guillain-Barré syndrome, CIDP, and necrotizing fasciitis. The primary conclusion was that during periods of IVIg shortage, there was a decrease in single-patient infusions. However, the total annual amount of IVIg used remained unchanged. The authors concluded that the decline in single infusions might have corresponded to less empiric use. During the sampling period in this study, IVIg in the U.S. market was in a period of stable supply. The PA process as described in this analysis is not intended to manage IVIg inventory.

The patterns of IVIg use in the United States are not well described in current published literature. Two key studies, similar to those from Canada, examined the patterns of IVIg use in hospital systems. Darabi et al. (2006) evaluated the use of IVIg at Massachusetts General Hospital for calendar year 2004 in a study of 194 patients. ${ }^{15}$ At the time of this study, IVIg required review against the hospital guidelines by a physician before it was authorized for patient use. The authors did not define these guidelines or describe the occurrence or criteria by which IVIg was denied-variables that would be of comparative interest with our current findings. The authors discovered that a limited number of diagnostic indications accounted for the majority of IVIg use. The highest use of IVIg was found in the treatment of chronic neuropathies-an indication with strong supporting evidence-representing 61.9\% of total grams infused.

An observational study by Chen et al. (2000) of IVIg use in 12 U.S. academic centers in 1996 assessed the inpatient use of IVIg in 251 patients. ${ }^{16}$ The authors also evaluated the FDA label status and supporting evidence for requests using the 1995 United Health Consortium guidelines. The study found that $43 \%$ of IVIg use was for labeled indications. Applying current FDA approvals to these data, which now include CIDP and multifocal motor neuropathy, would increase the usage to $47 \%$, since only a small cohort of patients with those additional diagnoses was identified. Of the $130(52 \%)$ patients who received IVIg for an off-label indication, $27 \%$ were in a "currently not 
Descriptive Review and Analysis of Immunoglobulin Utilization Management from 2,548 Prior Authorization Requests

\section{TABLE 5 Benefit Coverage Outcomes} by Age Group

\begin{tabular}{l|c|c|c}
\hline Age Group (Years) & Approved (n) & Denied (n) & Total \\
\hline$\leq 2$ & 20 & 1 & 21 \\
\hline $2-5$ & 48 & 10 & 58 \\
\hline $6-12$ & 121 & 22 & 143 \\
\hline $13-18$ & 81 & 5 & 86 \\
\hline $19-44$ & 575 & 130 & 705 \\
\hline $45-64$ & 1,066 & 252 & 1,318 \\
\hline $65-79$ & 161 & 28 & 189 \\
\hline$\geq 80$ & 12 & 4 & 16 \\
\hline Unknown & 10 & 2 & 12 \\
\hline Total & 2,094 & 454 & 2,548 \\
\hline
\end{tabular}

supported" group, based on the more recent guidelines presented here. ${ }^{16}$ In comparison with the other studies, the use of IVIg for treating neurologic conditions in this study was notably less ( $16.7 \%$ of patients). Chen et al. had one of the oldest sampling periods referenced, and the use of IVIg as a treatment intervention was likely less recognized at that time.

Our study provides additional details regarding the patterns of IVIg use by describing managed care benefit determinations. The percentages of benefit denials in this study for "supported" conditions suggest that many of the requests did not fulfill the requirements of the health plan's criteria, such as confirmation of diagnosis through submitted testing, inclusion of a patient history, and demonstrated failure of lower cost first-line interventions when applicable. Such determinations would require the health plans' reviewing teams to have expert knowledge of these conditions and their treatment. Only $53.6 \%$ of the indications identified in our sampled requests have been reviewed by the consensus studies cited in this analysis, stressing the need for evidence in the form of patient case reports and controlled trials.

A concerning finding in our study was the number of observed benefit denials for immunology conditions. A total of 166 denials were observed for conditions with "supported" and "conditional" evidence grades. Assuming that the diagnosis and ICD-9-CM coding by the requesting physicians were accurate, the costs of leaving these patients untreated is likely substantial. The aggregate annual costs of untreated primary immunodeficiencies per patient have been estimated to be $\$ 138,760$ (Table 6), which includes factors such as infections, antibiotic use, hospitalizations, and school/work days missed. ${ }^{1}$ Costs after start of treatment, which include Ig replacement (approximately $\$ 30,000^{1}$ ), are estimated at $\$ 60,297$ annually, resulting in a savings of $\$ 78,166$ per patient when compared with their untreated counterparts. ${ }^{1}$ According to these estimates, the 166 patients from our study who had a primary immune diagnosis and were denied benefit authorization may represent an annual economic burden of $\$ 23,034,160$. At
TABLE 6 Costs of the Most Frequent Conditions Affecting Patients with Primary Immunodeficiency (United States Only) ${ }^{1}$

\begin{tabular}{l|c|c|c}
\hline Condition & $\begin{array}{c}\text { Annual Cost } \\
\text { Per Patient } \\
\text { Before Start of } \\
\text { Treatment (\$) }\end{array}$ & $\begin{array}{c}\text { Annual Cost } \\
\text { Per Patient } \\
\text { After Start of } \\
\text { Treatment (\$) }\end{array}$ & $\begin{array}{c}\text { Annual } \\
\text { Savings Per } \\
\text { Patient (\$) }\end{array}$ \\
\hline Acute infections & 25,299 & 7,115 & 18,184 \\
\hline Severe infections & 34,299 & 4,588 & 29,641 \\
\hline Bacterial pneumonias & 28,249 & 6,053 & 22,196 \\
\hline Chronic infections & 2,175 & 612 & 1,562 \\
\hline $\begin{array}{l}\text { Physician/hospital/ } \\
\text { emergency room visits }\end{array}$ & 11,875 & 1,977 & 9,899 \\
\hline Hospitalizations & 29,792 & 7,913 & 21,880 \\
\hline Use of antibiotics & 946 & 414 & 532 \\
\hline School/work days missed & 6,195 & 1,625 & 4,569 \\
\hline Totals per patient & 138,760 & 30,297 & 108,462 \\
\hline
\end{tabular}

$\$ 10,009,302$, the notably lower cost of treatment with Ig would represent an annual avoided cost of $\$ 13,024,858$.

Immune deficiencies comprised the highest use in our study. Given the wide spectrum of immune deficiency disease, a health plan may have difficulty in determining the necessity for Ig therapy. Objective measures of immune system function can be obtained through such diagnostic means as vaccine response, total immunoglobulin levels, immunoglobulin isotypes, and subclass levels that can be referenced to determine whether Ig replacement is warranted. ${ }^{17-21}$ The patient's detailed history of infections is also essential. Patient-specific criteria require clinical interpretation and often do not fit well into algorithms derived for the purpose of determining insurance approval. A strong clinical history may well demonstrate extraordinary circumstances that warrant consideration of Ig for one patient but that may not apply to another patient with similar laboratory findings.

\section{Limitations}

Our study has several important limitations. First, the data may not have been representative of all PA requests received by the health plans in the sample period. The data were also geographically constrained to the Central and Northeast regions and thus may represent regional practice patterns. The assessments of supporting levels of clinical evidence did not consider the severity or stage of the diseases targeted by Ig treatment, nor other clinical factors that may have influenced treatment decisions. Patient charts were not available for this review. As a result, the accuracy of patient diagnoses could not be verified, nor could patient response to Ig treatment for any of the diagnoses under review be determined.

\section{Conclusions}

The patterns of use found in our analysis identified a significant portion of Ig requests for "supported" diagnoses that were 
denied by the health plans; this denial may restrict usage in patients who could benefit from therapy. A study to follow up on the medical costs associated with this population of patients would provide important data on the risks of nontreatment for "supported" diagnoses. We observed a total of 181 diagnostic indications. Of these, only 97 have been reviewed by expert consensus, leaving the burden on the health plan to research available medical evidence for the remaining indications-a time-consuming process with the potential for inconsistent interpretation. ${ }^{4}$ We found that only a small percentage of total requests were for indications with a current lack of consensus. An unexpected outcome was the high percentage of these cases that ultimately were authorized. We also note, as discussed in our limitations, that we may not have been presented with all of the requests in this category that were received by the health plan and that we were unable to ascertain how patients responded to Ig treatment for any diagnosis.

Immunoglobulin is a costly therapy, and for many conditions, long-term or lifelong treatment is needed. The estimated costs of untreated primary immune conditions may justify qualifying Ig therapy for these patients. The growing number of indications for Ig requires that health plans consider strategies to adapt to the current state of evidence and to review cases for best evidence prior to making an authorization decision.

Service providers, including specialty pharmacies and physicians, have a role in not only providing case evidence for the PA process, but also in providing literature through case reports that enhance the body of knowledge surrounding empiric therapy. Collectively, and as individual providers, specialty pharmacies collect thousands of patient experiences annually across a wide range of diagnostic indications. These pharmacies also collect data on long-term use that can play a role in supporting new indications. Many rare indications for IVIg may not receive a benefits approval based on a current lack of supporting evidence, while pharmacy providers may have data to support duration, time to effect, and overall effectiveness of therapy. Specialty pharmacy providers can partner with payers to implement programs to monitor use and also to provide evidence of therapeutic benefit that is lacking for many indications.

\section{Authors}

BRIAN TONKOVIC, PharmD, is Director of Therapy Management, and LINDA K. RUTISHAUSER, RN, is Therapy Management Analyst, EyeOn Therapy Management, Coram Specialty Infusion Services, Mount Prospect, Illinois.

AUTHOR CORRESPONDENCE: Brian Tonkovic, PharmD, EyeOn Therapy Management, Coram Specialty Infusion Services, 1471 Business Center Dr., Ste. 500, Mount Prospect, IL 60056. Tel.: 847.553.2590; Fax: 847.553.2750; E-mail: tonkovicb@coramhc.com.

\section{DISCLOSURES}

Tonkovic and Rutishauer worked on this article as part of regular employment. The authors report no other financial conflict of interest.

\section{REFERENCES}

1. Modell V, Gee B, Lewis DB, et al. Global study of primary immunodeficiency diseases (PI) — diagnosis, treatment, and economic impact: an updated report from the Jeffrey Modell Foundation. Immunol Res. 2011;51(1):61-70.

2. U.S. Food and Drug Administration. Vaccines, blood, and biologics: immune globulin intravenous (IGIV) indications. June 10, 2013. Available at: http://www.fda.gov/BiologicsBloodVaccines/BloodBloodProducts/ ApprovedProducts/LicensedProductsBLAs/FractionatedPlasmaProducts/ ucm133691.htm. Accessed December 30, 2013.

3. U.S. Department of Health and Human Services. Analysis of supply, distribution, demand, and access issues associated with immune globulin intravenous (IGIV): final report. February 2007. Available at: http://aspe. hhs.gov/sp/reports/2007/IGIV/report.pdf. Accessed December 30, 2013.

4. Leong H, Stachnik J, Bonk ME, Matuszewski, KA. Unlabeled uses of intravenous immune globulin. Am J Health Syst Pharm. 2008;65(19):1815-24.

5. EMD Serono. Managed care strategies for specialty pharmaceuticals. EMD Serono Specialty Digest, 9 th ed. Available at: http://specialtydigest.emdserono.com/Digest.aspx. Accessed December 30, 2013.

6. Feasby T, Banwell B, Benstead T, et al. Guidelines on the use of intravenous immune globulin for neurologic conditions. Transfus Med Rev. 2007;21(2 Suppl 1):S57-107.

7. Donofrio PD, Berger A, Brannagan TH 3rd, et al. Consensus statement: the use of intravenous immunoglobulin in the treatment of neuromuscular conditions report of the AANEM Ad Hoc Committee. Muscle Nerve. 2009;40(5):890-900. Available at: http://www.aanem.org/ getmedia/0b0cc37c-55f4-4d9d-b4e7-ecbcec01la5d/IVIG_CS_2009.pdf. aspx. Accessed December 31, 2013.

8. Skeie GO, Apostolski S, Evoli A, et al. Guidelines for treatment of autoimmune neuromuscular transmission disorders. Eur J Neurol. 2010;17(7):893-902. Available at: http://www.efns.org/fileadmin/user_upload/CME_articles/ CME_article_2010_July.pdf. Accessed December 31, 2013.

9. Anderson D, Ali K, Blanchette V, et al. Guidelines on the use of intravenous immune globulin for hematologic conditions. Transfus Med Rev. 2007;21(2 Suppl 1):S9-S56.

10. Elovaara I, Apostolski S, Van Doorn P, et al. EFNS guidelines for the use of intravenous immunoglobulin in treatment of neurological diseases: EFNS task force on the use of intravenous immunoglobulin in treatment of neurological diseases. Eur J Neurol. 2008;15(9):893-908. Available at: \%20http:/onlinelibrary.wiley.com/doi/10.1111/j.1468-1331.2008.02246.x/ pdf. Accessed December 31, 2013.

11. Orange JS, Hossny EM, Weiler CR, et al. Use of intravenous immunoglobulin in human disease: a review of evidence by members of the Primary Immunodeficiency Committee of the American Academy of Allergy, Asthma and Immunology. J Allergy Clin Immunol. 2006;117(4 Suppl):S525-S53. Available at: http://www.aaaai.org/Aaaai/media/MediaLibrary/PDF\%20 Documents/Practice\%20Resources/2006_IVIg_evidence_review.pdf. Accessed December 31, 2013

12. Shehata N, Palda VA, Meyer RM, et al. The use of immunoglobulin therapy for patients undergoing solid organ transplantation: an evidence-based practice guideline. Transfus Med Rev. 2010;24(Suppl 1):S7-S27.

13. Hanna K, Poulin-Costello M, Preston M, Maresky N. Intravenous immune globulin use in Canada. Can J Clin Pharmacol. 2003;10(1):11-16.

14. Pendergrast JM, Sher GD, Callum JL. Changes in intravenous immunoglobulin prescribing patterns during a period of severe product shortages, 1995-2000. Vox Sang. 2005;89(3):150-60. 
15. Darabi K, Abdel-Wahab O, Dzik WH. Current usage of intravenous immune globulin and the rationale behind it: the Massachusetts General Hospital data and a review of the literature. Transfusion. 2006;46(5):741-53.

16. Chen C, Danekas LH, Ratko TA, Vlasses PH, Matuszewski KA. A multicenter drug use surveillance of intravenous immunoglobulin utilization in U.S. academic health centers. Ann Pharmacother. 2000;34(3):295-99.

17. Weiler CR, Bankers-Fulbright JL. Common variable immunodeficiency: test indications and interpretations. Mayo Clin Proc. 2005;80(9):1187-200.

18. Agarwal S, Cunningham-Rundles C. Assessment and clinical interpretation of reduced IgG values. Ann Allergy Asthma Immunol. 2007;99(3):281-83. Available at: http://www.ncbi.nlm.nih.gov/pmc/articles/PMC3099256/pdf/ nihms293635.pdf. Accessed December 31, 2013.
19. Berger M. Principles of and advances in immunoglobulin replacement therapy for primary immunodeficiency. Immunol Allergy Clin North Am. 2008;28(2):413-37.

20. Bonilla FA, Bernstein IL, Kahn DA, et al. Practice parameter for the diagnosis and management of primary immunodeficiency. Ann Allergy Asthma Immunol. 2005;94(5 Suppl 1):S1-S63. Available at: http://www.aaaai. org/Aaaai/media/MediaLibrary/PDF\%20Documents/Practice\%20and\%20 Parameters/immunodeficiency2005.pdf. Accessed December 31, 2013.

21. Orange JS, Ballow M, Stiehm ER, et al. Use and interpretation of diagnostic vaccination in primary immunodeficiency: a working group report of the Basic and Clinical Immunology Interest Section of the American Academy of Allergy, Asthma and Immunology. J Allergy Clin Immunol. 2012;130(3 Suppl):S1-S24. 
APPENDIX ICD-9-CM Diagnostic Codes: Primary Immunodeficiency

\begin{tabular}{|c|c|}
\hline 279.00 & $\begin{array}{l}\text { Hypogammaglobulinemia, unspecified } \\
\text { • Agammaglobulinemia NOS }\end{array}$ \\
\hline 279.01 & Selective IgA immunodeficiency \\
\hline 279.02 & Selective IgM immunodeficiency \\
\hline 279.03 & $\begin{array}{l}\text { Other selective immunodeficiencies } \\
\text { - Selective deficiency of IgG }\end{array}$ \\
\hline 279.04 & $\begin{array}{l}\text { Congenital hypogammaglobulinemia } \\
\text { - Bruton's type } \\
\text { - X-linked }\end{array}$ \\
\hline 279.05 & $\begin{array}{l}\text { Immunodeficiency with increased IgM } \\
\text { - Autosomal recessive } \\
\text { - X-linked }\end{array}$ \\
\hline 279.06 & $\begin{array}{l}\text { Common variable immunodeficiency } \\
\text { • Dysgammaglobulinemia (acquired/congenital/primary) }\end{array}$ \\
\hline 279.09 & $\begin{array}{l}\text { Other } \\
\text { • Transient hypogammaglobulinemia of infancy }\end{array}$ \\
\hline 279.10 & Immunodeficiency with predominant T-cell defect, unspecified \\
\hline 279.11 & DiGeorge syndrome \\
\hline 279.12 & Wiskott-Aldrich syndrome \\
\hline 279.13 & Nezelof's syndrome \\
\hline
\end{tabular}

